\title{
Functionalization of Graphene with Nitrile Groups by Cycloaddition of Tetracyanoethylene Oxide
}

\author{
Xiaojun Peng, Yang Li, Guoliang Zhang, Fengbao Zhang, and Xiaobin Fan \\ State Key Laboratory of Chemical Engineering, Key Laboratory for Green Chemical Technology, \\ School of Chemical Engineering \& Technology, Tianjin University, Tianjin 300072, China \\ Correspondence should be addressed to Xiaobin Fan; xiaobinfan@tju.edu.cn \\ Received 12 March 2013; Accepted 16 September 2013 \\ Academic Editor: Sulin Zhang \\ Copyright (C) 2013 Xiaojun Peng et al. This is an open access article distributed under the Creative Commons Attribution License, \\ which permits unrestricted use, distribution, and reproduction in any medium, provided the original work is properly cited. \\ Graphene has got considerable attention in both experimental and theoretical fields for its extraordinary properties. Covalent \\ functionalization is an efficient strategy to render graphene additional properties and overcome its shortcomings such as zero \\ band gap and nondispersibility in solvents. This study reports the synthesis and characterizations of a new kind of functionalized \\ graphene, graphene-TCNEO, obtained by 1,3-dipolar cycloaddition. The graphene-TCNEO was systematically characterized by \\ FTIR, Raman, XPS, SEM, TEM, and EDS mapping, and the covalent linkage between graphene and tetracyanoethylene oxide was \\ firmly verified. Considering the great diversity of nitrile chemistry, the obtained graphene-TCNEO could be further transformed \\ into other graphene-based derivatives with interesting properties.
}

\section{Introduction}

Graphene has got considerable attention from both experimental and theoretical fields for its extraordinary properties, such as large surface area, unique mechanical, thermal, opti$\mathrm{cal}$, and electronic transport properties [1-3]. Although these properties endow graphene great potential in many practical applications, its inertness nature, zero band gap, and nondispersibility in solvents [4] weaken its competitive strength in the synthesis of composites, semiconductors, sensors, and so forth [5-8]. Therefore, functionalization of graphene [9] has attracted widespread attention in the past few years. Covalent functionalization [10-12] of graphene with organic atoms or molecules is of particular interest, as it can combine the unique properties of each component while overcoming the shortcomings of graphene as mentioned above. Through direct attachment of organic atoms or molecules to the $\mathrm{C}=\mathrm{C}$ bonds of graphene $[13,14]$ and the rich covalent reactions of oxygen-containing groups in graphene oxide [15-17], a variety of strategies to functionalize graphene for different application purposes have been demonstrated. In this study, we report a method to introduce abundant nitrile groups to pristine graphene by cycloaddition reaction of graphene with tetracyanoethylene oxide [18-20] (TCNEO) (Scheme 1).

\section{Experimental Details}

The process for the funcitionalized graphene material is shown in Scheme 1. The mixture of desired $10 \mathrm{mg}$ graphene prepared by the methods of high temperature expansion [21], $60 \mathrm{mg}$ TCNEO, and $200 \mathrm{~mL}$ solution of 1,2-dibromoethane were added into a $250 \mathrm{~mL}$ round-bottomed flask and stirred and heated at $140^{\circ} \mathrm{C}$ to reflux for $24 \mathrm{~h}$ under $\mathrm{N}_{2}$ atmosphere. After the reaction completed, the reaction mixture was filtered through nylon membrane. The obtained filter cake was washed several times by anhydrous ethanol and deionized water, respectively. The final product was suspended in deionized water through sonication and dried under vacuum freezedrying to obtain the desired grapheneTCNEO.

The graphene and functionalized graphene were characterized by infrared spectrum (FTIR) (Thermo-Nicolet 380), $\mathrm{X}$-ray photoelectron spectroscopy (XPS) (PerkinElmer, PHI 1600 spectrometer), Raman spectroscopy (NT-MDT NTEGRA Spectra), scanning electron microscopy (SEM) (Hitachi S4800), high-resolution transmission electron microscopy (HRTEM) (Philips Tecnai G2 F20), and energy dispersive spectroscopy (EDS) (Hitachi S4800). 


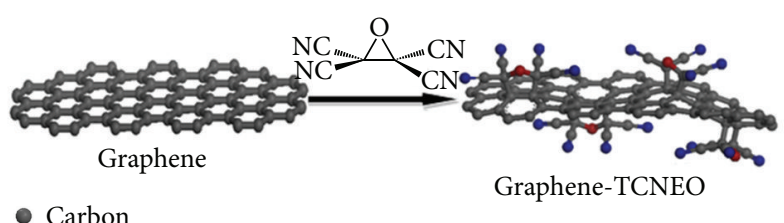

- Carbon

- Oxygen

SCHEME 1: Synthesis of grapheme-TCNEO hybrid materials.

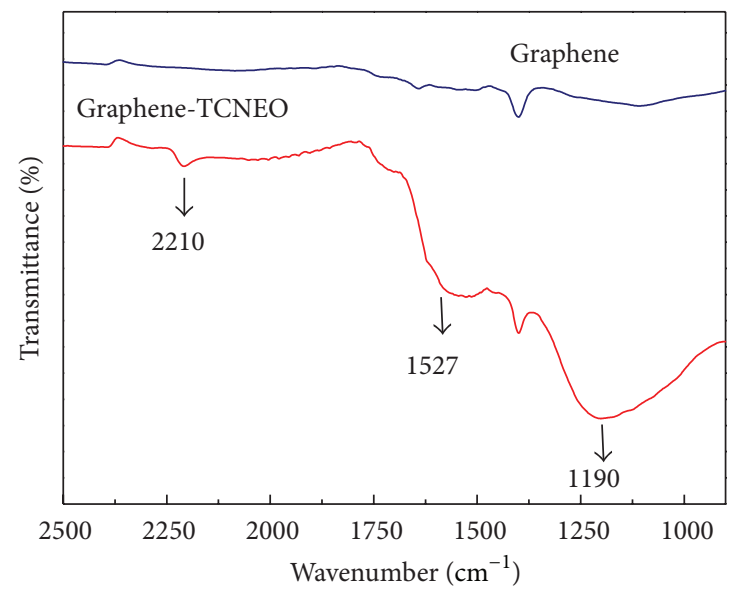

FIGURE 1: FTIR spectra of graphene and grapheme-TCNEO, the peaks located at the $1399 \mathrm{~cm}^{-1}$ by the residual of solvent in the purge process.

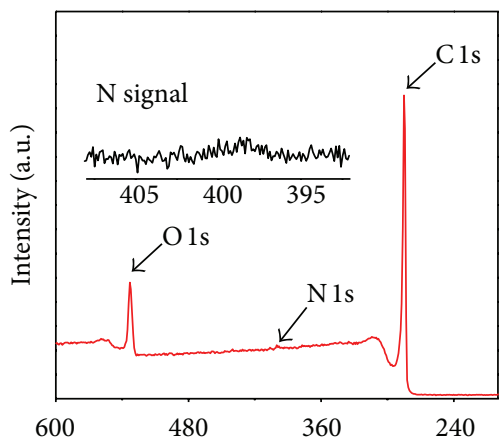

(a)

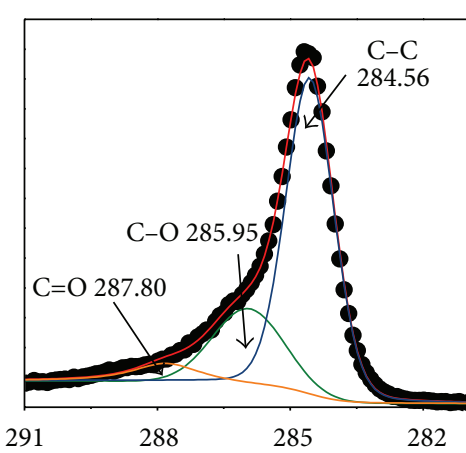

(d)

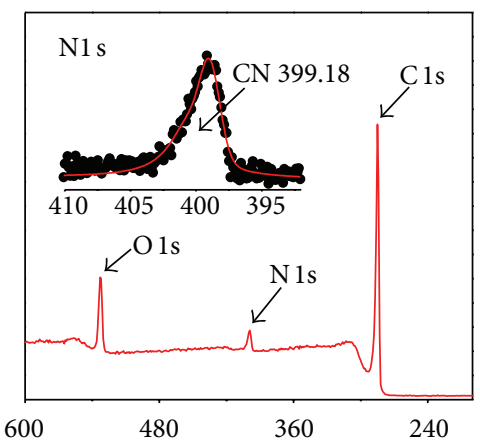

(b)

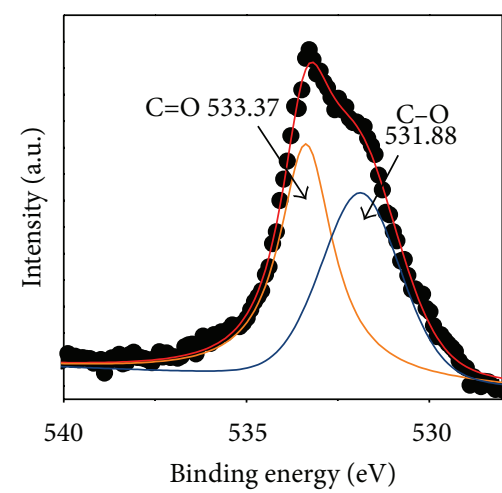

(e)

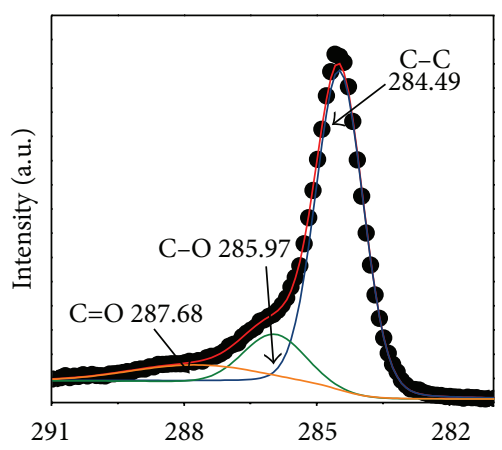

(c)

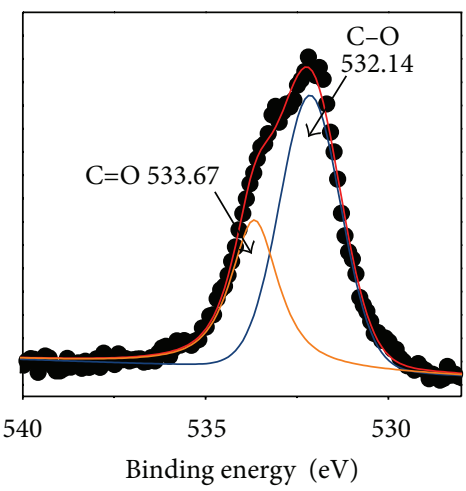

(f)

FIGURE 2: XPS general spectra and curve fit of $\mathrm{N}$ 1s core-level spectra for graphene (a) and functionalized graphene (b), C 1s core level for graphene (c) and functionalized graphene (d), and $\mathrm{O}$ 1s core level for graphene (e) and functionalized graphene (f). 


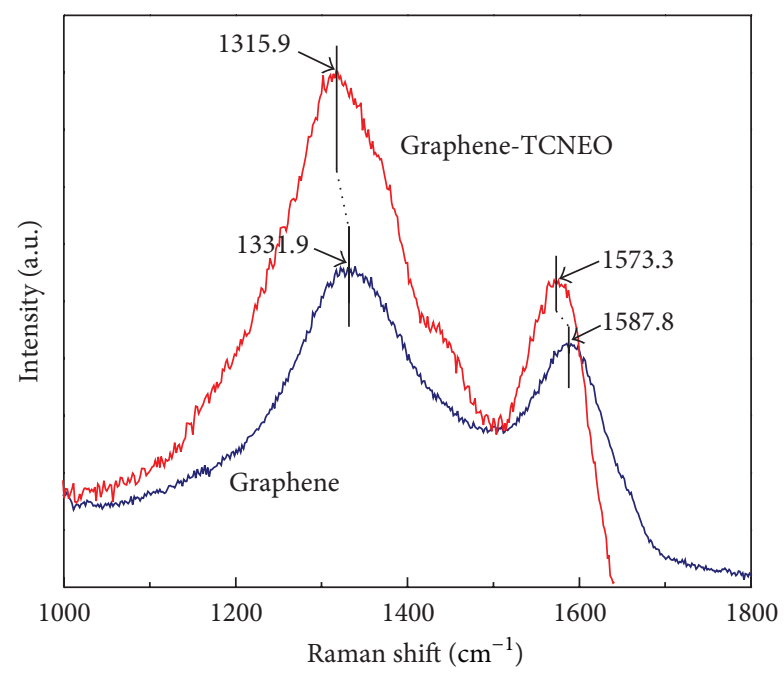

FIGURE 3: The Raman spectrum of graphene and grapheme-TCNEO.
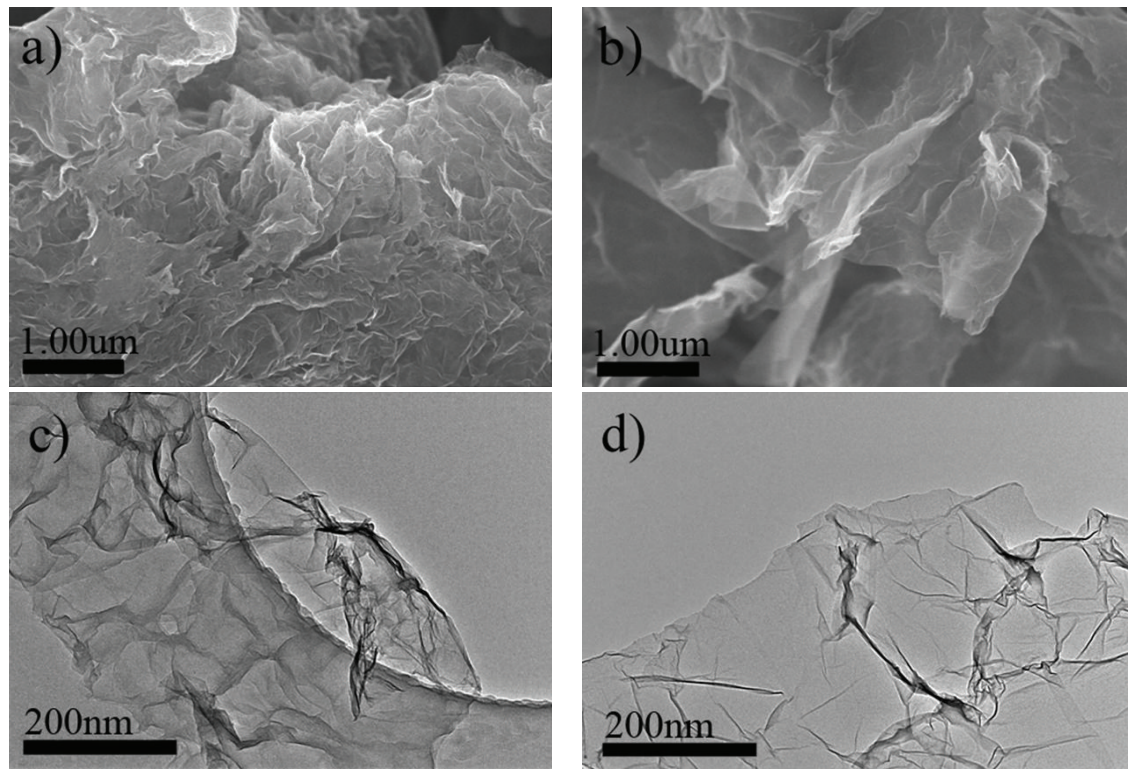

FIGURE 4: SEM images of graphene (a) and functionalized graphene (b), TEM images of graphene (c) and functionalized graphene (d).

\section{Results and Discussions}

Graphene and tetracyanoethylene oxide were mixed and dispersed in 1,2-dibromoethane, followed by continuous reactions at $140^{\circ} \mathrm{C}$ for $12 \mathrm{~h}$ in the $\mathrm{N}_{2}$ atmosphere. After being purified by repeated filtration and redispersion, the final product was obtained. As can be seen from Figure 1, the obtained grapheme-TCNEO shows the characteristic vibration of nitrile groups from TCNEO at $2210 \mathrm{~cm}^{-1}$. Because of the conjugation effect caused by the conjugated structure of the attached graphene sheets, the vibration of the nitrile groups shifts to relatively lower wavenumber [22, 23]. In addition, two new peaks at $1527 \mathrm{~cm}^{-1}$ and $1190 \mathrm{~cm}^{-1}$ are also readily observed in grapheme-TCNEO, respectively and represent the skeletal vibration of the generated polysubstituted homocyclic ring and the stretching vibration of the introduced $\mathrm{C}-\mathrm{O}$. These results will be further supported by later $\mathrm{X}$-ray photoelectron spectroscopy (XPS) and Raman analysis.

Compared with the graphene (Figure 2(a)), a new peak at about $399 \mathrm{eV}$ corresponding to the binding energy of $\mathrm{N}$ $1 \mathrm{~s}$ is clearly observed in the obtained grapheme-TCNEO, attributed to the introduced nitrile groups (Figure 2(b)). In the corresponding high-resolution $\mathrm{N}$ 1s spectrum, there is a single peak binding in $399.18 \mathrm{eV}$ that suggests the presence of nitrile groups [24]. Based on the elemental ratios, the degree of functionalization is calculated to be approximately 1 TCNEO group in 85 carbon atoms. Additional evidence for the introduction of TCNEO to graphene comes from the obvious increase of $\mathrm{C}-\mathrm{O}$ bonds in the $\mathrm{C}$ 1s and $\mathrm{O}$ 1s spectra of the obtained grapheme-TCNEO (Figures 2(c)-2(f)). 

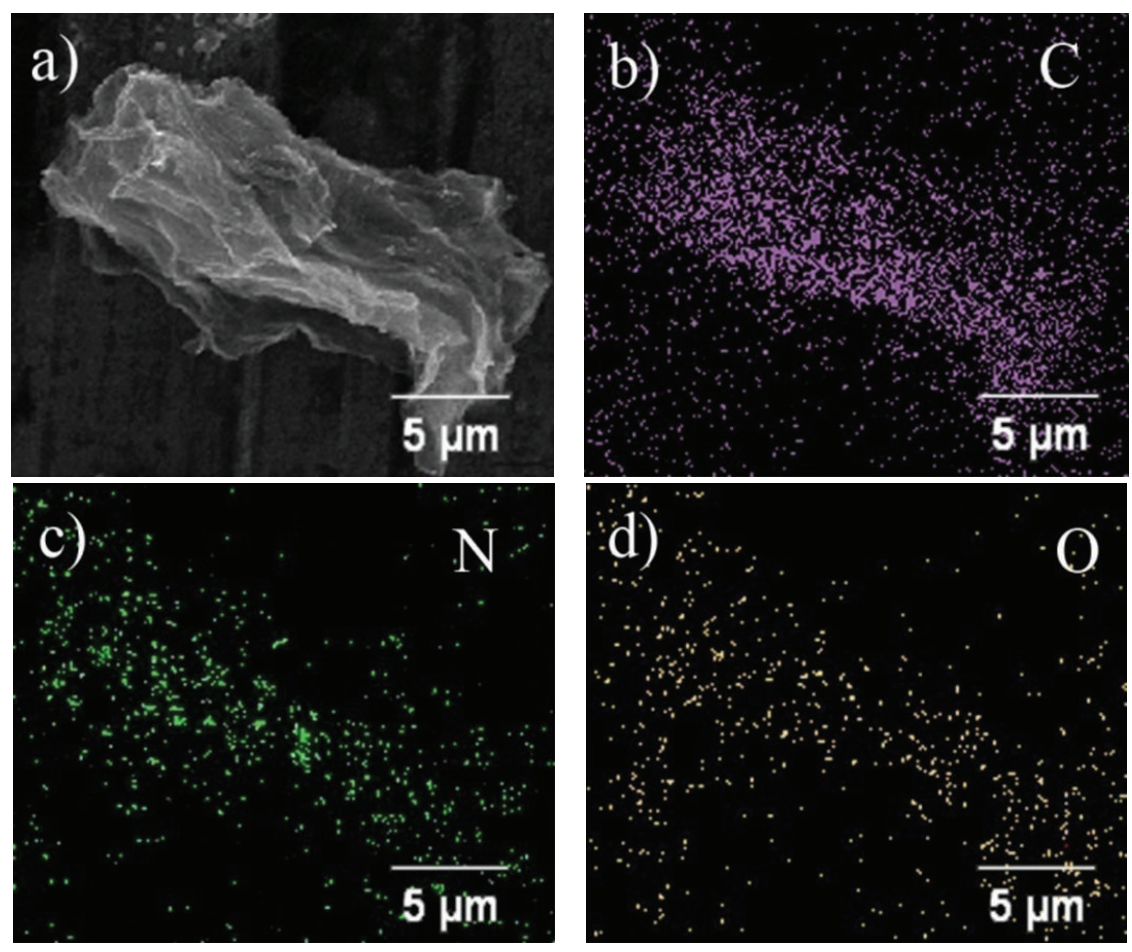

FIGURE 5: The SEM image of the grapheme-TCNEO (a) and corresponding quantitative EDS element mapping of C (b), N (c), and O (d).

Raman spectra analysis [25] reveals a significant increase in the intensity ratio $\left(I_{\mathrm{D}} / I_{\mathrm{G}}\right)$ of graphene after functionalization (Figure 3). Considering the $\mathrm{G}$ band and $\mathrm{D}$ band that represent the $\mathrm{sp}^{2} \mathrm{C}$ atoms and defects, respectively, it can be anticipated that the destructions of the $\mathrm{sp}^{2}$ hybridization structure are raised from the successful cycloaddition reactions. This result matches up with the results of FTIR and XPS discussed above. Moreover, the noticeable red shifts $\left(15 \mathrm{~cm}^{-1}\right)$ of the $\mathrm{G}$ band and $\mathrm{D}$ band are observed after the reaction, probably due to the introduced conjugated nitrile groups that alter the conjugate structure of the graphene basal plane.

The structural change of graphene before and after functionalizations was further investigated by scanning electron microscope (SEM) and transmission electron microscope (TEM). As can be seen from Figures 4(a) and 4(b), the obtained grapheme-TCNEO shows a wrinkled structure similar to the pristine graphene sheets. TEM images (Figures 4(c) and 4(d)) demonstrate that both the grapheme-TCNEO and graphene are semitransparent due to their thin natures and have the dark veins that simply resulted from overlapped regions of the graphene sheets. The corresponding quantitative energy dispersive X-ray spectroscopy (EDS) mappings (Figure 5) confirm that elements $\mathrm{C}, \mathrm{N}$, and $\mathrm{O}$ are homogeneously distributed on the surfaces of the obtained grapheme-TCNEO, providing visualized evidence for the successful functionalization of graphene with nitrile groups.

\section{Conclusions}

In summary, we report an efficient strategy to functionalize graphene with nitrile groups. Systematic characterizations demonstrated that the nitrile groups were homogeneously introduced to the surfaces of graphene via the cycloaddition reaction with tetracyanoethylene oxide (TCNEO). Considering the diversity of nitrile chemistry, the obtained grapheneTCNEO may be further transformed into other graphenebased derivatives with interesting properties.

\section{Acknowledgments}

This study was supported by the National Natural Science Funds for Excellent Young Scholars (no. 21222608), Research Fund of the National Natural Science Foundation of China (no. 21106099), Foundation for the Author of National Excellent Doctoral Dissertation of China (no. 201251), the Tianjin Natural Science Foundation (no. 11JCYBJC01700), and the Programme of Introducing Talents of Discipline to Universities (no. B06006).

\section{References}

[1] A. Peigney, C. Laurent, E. Flahaut, R. R. Bacsa, and A. Rousset, "Specific surface area of carbon nanotubes and bundles of carbon nanotubes," Carbon, vol. 39, no. 4, pp. 507-514, 2001.

[2] C. Lee, X. Wei, J. W. Kysar, and J. Hone, "Measurement of the elastic properties and intrinsic strength of monolayer graphene," Science, vol. 321, no. 5887, pp. 385-388, 2008.

[3] K. S. Novoselov, A. K. Geim, S. V. Morozov et al., "Electric field in atomically thin carbon films," Science, vol. 306, no. 5696, pp. 666-669, 2004.

[4] J. C. Meyer, A. K. Geim, M. I. Katsnelson, K. S. Novoselov, T. J. Booth, and S. Roth, "The structure of suspended graphene sheets," Nature, vol. 446, no. 7131, pp. 60-63, 2007. 
[5] H. Bai, C. Li, and G. Q. Shi, "Functional composite materials based on chemically converted graphene," Advanced Materials, vol. 23, no. 9, pp. 1089-1115, 2011.

[6] P. K. Santra and P. V. Kamat, "Mn-doped quantum dot sensitized solar cells: a strategy to boost efficiency over 5\%," Journal of the American Chemical Society, vol. 134, no. 5, pp. 2508-2511, 2012.

[7] O. Akhavan, E. Ghaderi, and R. Rahighi, "Toward single-DNA electrochemical biosensing by graphene nanowalls," ACS Nano, vol. 6, no. 4, pp. 2904-2916, 2012.

[8] J. G. Yu, M. Jaroniec, H. G. Yu, and W. H. Fan, "Synthesis, characterization, properties, and applications of nanosized photocatalytic materials," Journal of Nanomaterials, vol. 2012, Article ID 783686, 3 pages, 2012.

[9] N. Gorjizadeh and Y. Kawazoe, "Chemical functionalization of graphene nanoribbons," Journal of Nanomaterials, vol. 2010, Article ID 513501, 7 pages, 2010.

[10] H. F. Yang, C. S. Shan, F. Li, D. X. Han, Q. X. Zhang, and L. Niu, "Covalent functionalization of polydisperse chemicallyconverted graphene sheets with amine-terminated ionic liquid," Chemical Communications, vol. 26, pp. 3880-3882, 2009.

[11] J. R. Lomeda, C. D. Doyle, D. V. Kosynkin, W. F. Hwang, and J. M. Tour, "Diazonium functionalization of surfactant-wrapped chemically converted graphene sheets," Journal of the American Chemical Society, vol. 130, no. 48, pp. 16201-16206, 2008.

[12] J. Y. Ji, G. H. Zhang, H. Y. Chen et al., "A general strategy to prepare graphene-metal/metal oxide nanohybrids," Journal of Materials Chemistry, vol. 21, no. 38, pp. 14498-14501, 2011.

[13] L. H. Liu, M. M. Lerner, and M. D. Yan, "Derivitization of pristine graphene with well-defined chemical functionalities," Nano Letters, vol. 10, no. 9, pp. 3754-3756, 2010.

[14] X. Y. Zhang, L. L. Hou, A. Cnossen et al., "One-pot functionalization of graphene with porphyrin through cycloaddition reactions," Chemistry - A European Journal, vol. 17, no. 32, pp. 8957-8964, 2011.

[15] Y. Li, H. Y. Chen, L. Y. Voo et al., "Synthesis of partially hydrogenated graphene and brominated graphene," Journal of Materials Chemistry, vol. 22, no. 30, pp. 15021-15024, 2012.

[16] S. Stankovich, R. D. Piner, S. T. Nguyen, and R. S. Ruoff, "Synthesis and exfoliation of isocyanate-treated graphene oxide nanoplatelets," Carbon, vol. 44, no. 15, pp. 3342-3347, 2006.

[17] Y. W. Zhu, S. Murali, W. W. Cai et al., "Graphene and graphene oxide: synthesis, properties, and applications," Advanced Materials, vol. 22, no. 35, pp. 3906-3924, 2010.

[18] W. J. Linn and R. E. Benson, "Tetracyanoethylene oxide. II. Addition to olefins, acetylenes, and aromatics," Journal of the American Chemical Society, vol. 87, no. 16, pp. 3657-3665, 1965.

[19] W. J. Linn, "Tetracyanoethylene oxide. III. Mechanism of the addition to olefins," Journal of the American Chemical Society, vol. 87, no. 16, pp. 3665-3672, 1965.

[20] K. C. Nicolaou, S. A. Snyder, T. Montagnon, and G. Vassilikogiannakis, "The diels-alder reaction in total synthesis," Angewandte Chemie, vol. 41, no. 10, pp. 1668-1698, 2001.

[21] P. Guo, H. H. Song, and X. H. Chen, "Electrochemical performance of graphene nanosheets as anode material for lithiumion batteries," Electrochemistry Communications, vol. 11, no. 6, pp. 1320-1324, 2009.

[22] R. E. del Sesto, J. S. Miller, P. Lafuente, and J. J. Novoa, "Exceptionally Long (> or $=2.9$ A) CC bonding interactions in pi-[TCNE]2(2-) dimers: two-electron four-center cationmediated CC bonding interactions involving pi* electrons," Chemistry-A European Journal, vol. 8, no. 21, pp. 4894-4908, 2002.
[23] B. A. Lindquist and S. A. Corcelli, "Nitrile groups as vibrational probes: calculations of the $\mathrm{C} \equiv \mathrm{N}$ infrared absorption line shape of acetonitrile in water and tetrahydrofuran," Journal of Physical Chemistry B, vol. 112, no. 20, pp. 6301-6303, 2008.

[24] D. Cagniant, R. Gruber, J. P. Boudou, C. Bilem, J. Bimer, and P. D. Salbut, "Structural characterization of nitrogen-enriched coals," Energy and Fuels, vol. 12, no. 4, pp. 672-681, 1998.

[25] S. Niyogi, E. Bekyarova, M. E. Itkis et al., "Spectroscopy of covalently functionalized graphene," Nano Letters, vol. 10, no. 10, pp. 4061-4066, 2010. 

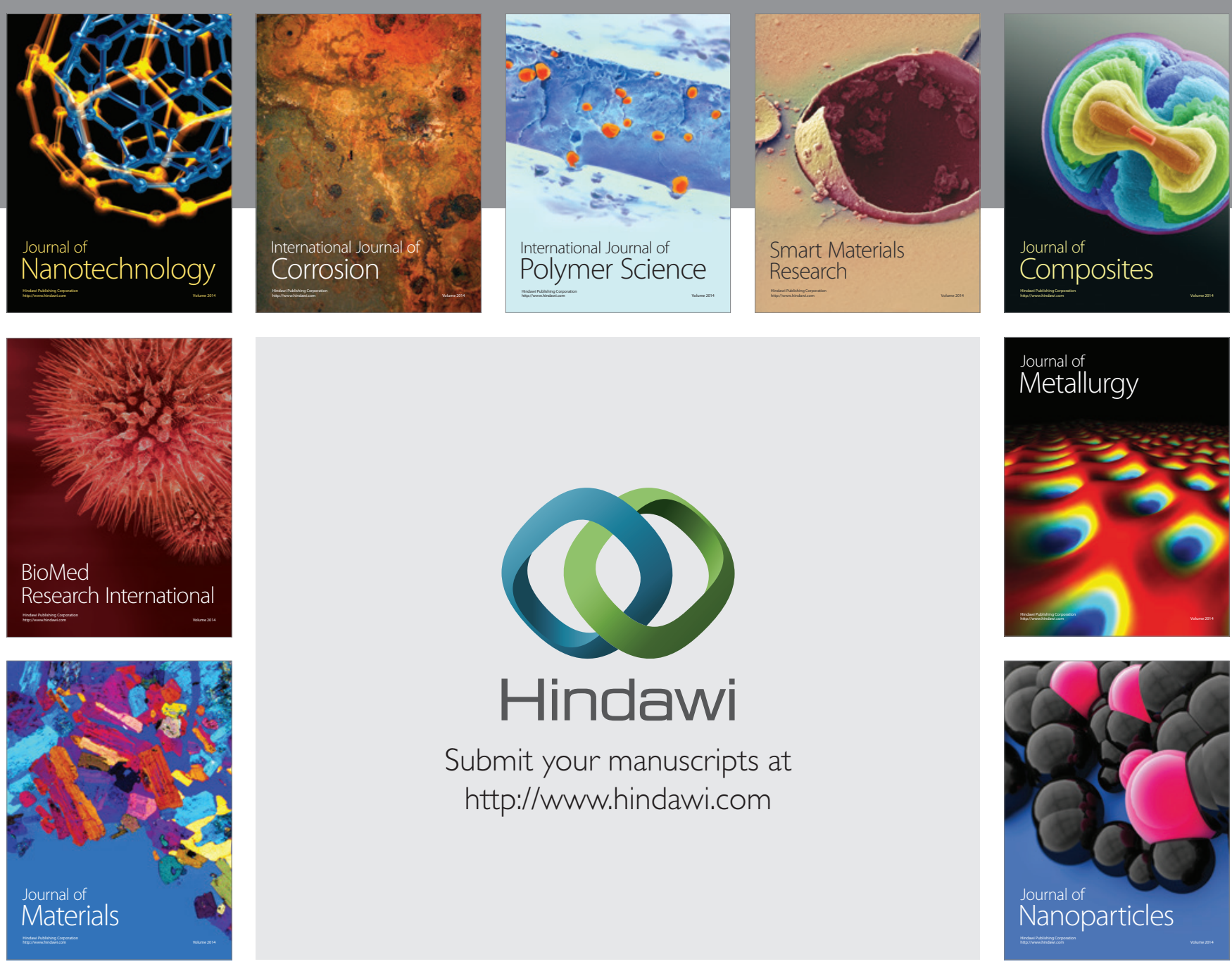

Submit your manuscripts at http://www.hindawi.com
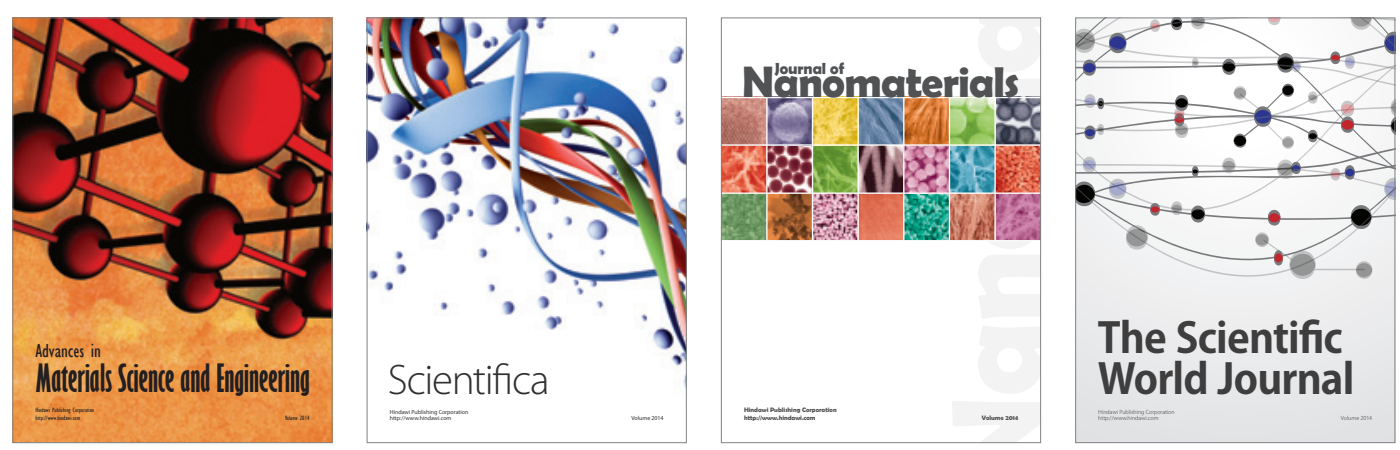

\section{The Scientific World Journal}
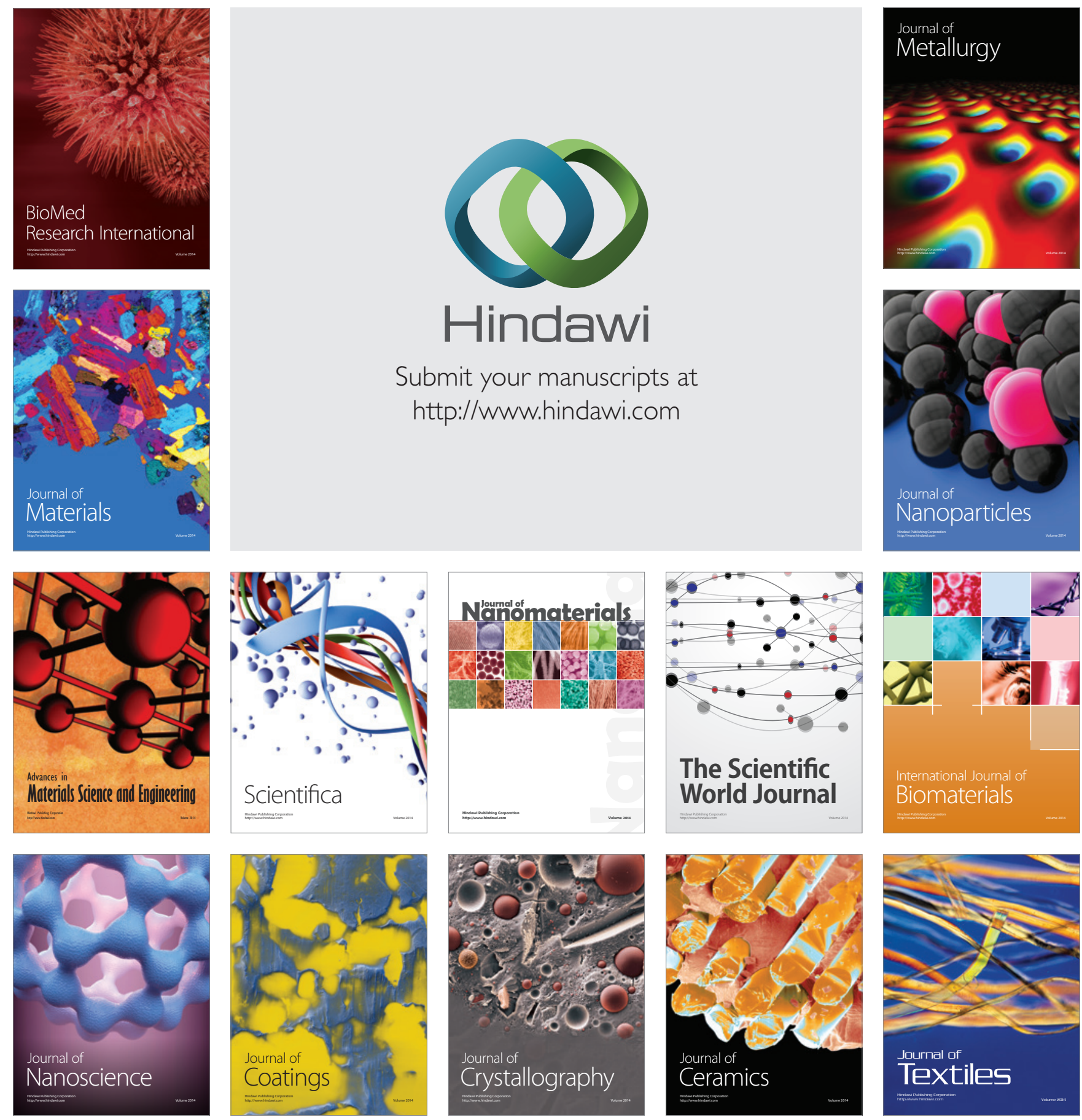